\title{
Maximum Likelihood Estimation of Time Delay for First Order Linear System
}

\author{
Jie Su, Hui Lu* and Xuguang Wang \\ North China Electric Power University, Baoding 071003, P. R. China \\ ${ }^{*}$ Corresponding author
}

\begin{abstract}
In the field of industrial control, the dynamic mathematical model of the main equipment or process of the system is established by the field data, which has become the hot spot of system identification at present. Due to various reasons from the industrial production process and means of measuring, the time delay is usually unavoidable in the process control system, if the phenomenon of time delay is not paid attention, which may lead to a sluggish response or even trigger instability. In this paper, maximum likelihood estimation of time delay is proposed for first order linear single-input-single-output (SISO) time delay system to estimate the time delay parameter. Comparative simulation experiments are provided to verify the justifiability and effectiveness of the proposed method.
\end{abstract}

Keywords-time delay estimation; MLE; copula; parameter identification

\section{INTRODUCTION}

Time delay is usually unavoidable in control systems, if the phenomenon of time delay is not paid attention, which may lead to a sluggish response or even trigger instability ${ }^{[1]}$. Therefore, the time delay estimation (TDE) draws more and more attention and becomes one of the most important topics in the field of system identification.

In recent years, various methods for TDE have been reported in the literature. According to the target source and the detection system, the time delay estimation problem can be divided into two types: active time delay estimation and passive time delay estimation $^{[2]}$. In the field of control and signal processing, most methods have been suggested for active time delay estimation, such as time-delay approximation methods, explicit time-delay parameter methods, area and moment methods, and higherorder statistics (HOS) methods. In time-delay approximation methods, the time delay is not an explicit parameter in the model. In [3], Kurz and Goedecke estimated time delay by measuring the time delay to the start (the beginning of the nonzero part) of an estimated impulse response of the system. Carter ${ }^{[4]}$ found the maximum of the cross-correlation between input and output, which was a common method. In [5], Horch and Isaksson studied the phase of the discrete-time all-pass part (DAP methods). Bjöklund and Ljung ${ }^{[6]}$ found that DAP methods could fail completely in some cases. The reason was that the noise moving zeros across the unit circle. They proposed an improved method named "zero guarding" to make DAP methods more robust to noise. In explicit time-delay parameter methods, the time delay is an explicit parameter to be estimated in the model. Estimating time delays via state-space identification methods, in which the delay model was selected with the lowest loss function ${ }^{[9]}$. Elnaggar et al. developed a variable regression estimation technique to provide direct delay estimation, which was suitable for estimating industrial engineering ${ }^{[10]}$. The modified area and moment method for the first order system was described to estimate the time delay, which avoided the second order moment integration ${ }^{[11]}$. Nikias and $\mathrm{Pan}^{[12]}$ proposed a time delay estimation method based on third-order statistics. The above methods estimate the time delay parameters based on the model structure of linear system. The model based methods always need a prior of the studied system, such as type of the system model, which limits the generalization of those methods.

In this paper, a method is proposed for first order linear single-input-single-output (SISO) time delay system to estimate the time delay parameter. We construct a likelihood function with the input and output data of the system and time delay parameter as independent variables, and obtain the time delay parameter by maximizing log-likelihood function of time delay.

\section{2-COPULA}

When the marginal distributions of multiple random variates are known, the coupling relationship between those random variates can be described by Copula, i.e. Copula connects the marginal distributions of the random variates to the joint distribution. This section states 2-Copula. See $[13,14]$ for more details.

\section{A. 2- Copula Definition}

2-copula is a function $\mathrm{C}:[0,1]^{2} \rightarrow[0,1]$ whose definition field is $[0,1]^{2}$ with the following properties:

(1) $\forall u, v \in[0,1], \mathrm{C}(u, 0)=\mathrm{C}(0, v)=0, \mathrm{C}(u, 1)=u$, $\mathrm{C}(1, v)=v$;

(2) $\forall u_{1}, u_{2}, v_{1}, v_{2} \in[0,1]$, when $u_{1}<u_{2}, v_{1}<v_{2}$ is $\mathrm{C}\left(u_{2}, v_{2}\right)-\mathrm{C}\left(u_{1}, v_{2}\right)-\mathrm{C}\left(u_{2}, v_{1}\right)+\mathrm{C}\left(u_{1}, v_{1}\right) \geq 0$.

The significance of the Copula is that it characterizes the dependent structure between multiple random variates. Suppose $X_{1}, X_{2}$ represent random vectors whose distribution functions are recorded as

$$
F\left(x_{1}, x_{2}\right)=P\left(X_{1} \leq x_{1}, X_{2} \leq x_{2}\right), \boldsymbol{x}=\left(x_{1}, x_{2}\right) \in R^{2}
$$

The marginal distributions of the random variates $X_{i}$ are recorded as

$$
F_{i}(x)=P\left(X_{i} \leq x\right), i=1,2 .
$$


According to theorem (Sklar) ${ }^{[15]}$, there is a unique 2-Copula $\mathrm{C}:[0,1]^{2} \rightarrow[0,1]$, for $\forall x \in R^{2}$,

$$
F\left(x_{1}, x_{2}\right)=P\left(X_{1} \leq x_{1}, X_{2} \leq x_{2}\right)=C\left(F_{1}\left(x_{1}\right), F_{1}\left(x_{2}\right)\right)
$$

It can be seen that the joint distribution of two random variables can be constructed through two separated steps, i.e. one step is the marginal distributions of the random variates, the other step is the dependent structure between the random variates, that is the Copula.

\section{B. 2-Copula Estimation}

When the Copula between random variates is unknown, it is necessary to estimate Copula in some way. In [16-21], various Copula estimation methods are introduced, which are roughly divided into two categories. The first category is a priori knowledge of the marginal distributions type of known random variables and the type of Copula. In this kind of problem, the undetermined parameters are estimated by maximum likelihood estimation and other methods (see e.g. $[18,20]$ ); The second category only knows the type of partial distribution (marginal distributions or joint distribution type) or has not prior knowledge of the distribution type. In this kind of problem, Copula can be estimated by empirical formula or several types of distribution are artificially designated as a priori, and the undetermined parameters are estimated through based on Bayesian method. Finally, it selects the final distribution type using some criteria such as AIC, BIC, DIC (see e.g. [17,22,23]).

In practical problems, if we do not know the distribution of random variates at all, we can get many observations of random variates. It is desirable to record the observed values of the random variates $X_{1}, X_{2}$ as a set $D_{j}=\left\{x_{j}^{i} \mid i=1,2, \ldots, n\right\}(j=$ $1,2)$. The empirical formula in the second category of Copula estimation method can be used, and the marginal distributions of the random variates are

$$
\tilde{F}_{j}(x)=\frac{1}{n} \sum_{i=1}^{n} 1\left(x_{j}^{i} \leq x\right), j=1,2 .
$$

Similarly, the Copula is estimated as

$$
\tilde{C}\left(u_{1}, u_{2}\right)=\frac{1}{n} \sum_{i=1}^{n} 1\left(\tilde{F}_{1}\left(x_{1}^{i}\right) \leq u_{1}, \tilde{F}_{2}\left(x_{2}^{i}\right) \leq u_{2}\right)
$$

\section{MAXimum LiKeliHOOd ESTIMATION OF TIME DELAY}

In this paper, we only discuss the time delay estimation problem between single input and single output system, because the time delay calculation problem between multiple input and multiple output system is equivalent to the time delay calculation between multiple single input and single output system.

Suppose $X_{1}, X_{2}$ are real value random variates, where the random variate $X_{1}$ is input and the random variate $X_{2}$ is output. The set of observations for the random variates $X_{1}, X_{2}$ at a fixed time $t_{0}$ are recorded as $D_{j}=\left\{X_{j}^{i}\right\}(\mathrm{j}=1,2)$; When $X_{1}, X_{2}$ delay time $k_{j} t_{0}\left(k_{j}\right.$ taken as an integer, $\left.\mathrm{j}=1,2\right)$, they are recorded as random variates $X_{1}\left[k_{1}\right], X_{2}\left[k_{2}\right]$, then the set of observations for the random variates $X_{1}\left[k_{1}\right], X_{2}\left[k_{2}\right]$ are recorded as $D_{j}\left[k_{j}\right]=\left\{X_{j}^{i+k_{j}}\right\}(\mathrm{j}=1,2)$. When the sampling data are enough, it can be assumed that $X_{1}, X_{2}$ and $X_{1}\left[k_{1}\right], X_{2}\left[k_{2}\right]$ have the same marginal distributions. The Copula of $X_{1}, X_{2}$ is not only related to the marginal distributions of $X_{1}, X_{2}$, but also related to the time delay between them. In general, if the time delay between $X_{1}, X_{2}$ is different, the Copula of $X_{1}, X_{2}$ is different. Thus, the Copula of $X_{1}, X_{2}$ can be represented as function form via their marginal distributions and time delay, i.e. $\mathrm{C}\left(F_{1}\left(X_{1}\right), F_{2}\left(X_{2}\right), k_{1}, k_{2}\right)$. Since the time delay between $X_{1}, X_{2}$ is relative time delay and the degree of freedom of $k_{1}, k_{2}$ is 1 , we can take $k_{1}=0$. Therefore, the time delay between $X_{1}, X_{2}$ is $k_{2}$. For the convenience of writing, we use $k$ instead of $k_{2}$ in the following equation.

The density of 2-Copula is

$$
c\left(u_{1}, u_{2}, k\right)=\frac{\partial C\left(u_{1}, u_{2}, k\right)}{\partial u_{1} \partial u_{2}} .
$$

According to theorem (Sklar) ${ }^{[15]}$, the density $\mathrm{f}$ of the 2dimensional distribution function $\mathrm{F}$ can be represented as

$$
f\left(\mathrm{x}_{1}, \mathrm{x}_{2}\right)=\mathrm{c}\left(F_{1}\left(X_{1}\right), F_{2}\left(X_{2}\right), k\right) f_{1}\left(\mathrm{x}_{1}\right) f_{2}\left(\mathrm{x}_{2}\right)
$$

where $f_{1}\left(\mathrm{x}_{1}\right)$ and $f_{2}\left(\mathrm{x}_{2}\right)$ represent the marginal probability density functions of the random variates $X_{1}, X_{2}$. The random vectors $\mathbf{X}=\left(X_{1}, X_{2}\right)$ are subjected to multiple independent samples to obtain their log-likelihood function

$$
L=\sum_{i} \log f\left(\mathrm{x}_{1}{ }^{i}, \mathrm{x}_{2}{ }^{i+k}\right)
$$

In order to be able to solve the impact of time delay on the system, we construct the following log-likelihood function

$$
L(k)=\sum_{i} \log f\left(\mathrm{x}_{1}{ }^{i}, \mathrm{x}_{2}{ }^{i+k}\right)
$$

According to the equation (7), $L$ can be decomposed into the following two parts

$$
L(k)=\underbrace{L_{c}}_{\text {dependence }}+\underbrace{\sum_{j=1}^{2} L_{j}}_{\text {marginals }},
$$

where

$$
L_{c}=\sum_{i} \log c\left(F\left(x_{1}^{i}\right), F\left(x_{2}{ }^{i+k}\right), k\right) .
$$

$L_{c}$ is the log-likelihood contribution in from dependence structure in data represented by the copula C. $L_{c}$ varies with the time delay $k ; L_{j}$ has the following form

$$
L_{j}=\left\{\begin{array}{cc}
\sum_{i} \log f_{1}\left(x_{1}{ }^{i}\right), & j=1 \\
\sum_{i} \log f_{2}\left(x_{2}{ }^{i+k}\right), & j=2
\end{array}\right.
$$

$L_{j}$ is the log-likelihood contributions from each margin: observe that $\sum_{j}^{2} L_{j}$ in (10) is exactly the log-likelihood of the sample under the independence assumption. When the sample 
are enough, the value of this part varies little with the time delay $k$. Theoretically, with taking the partial derivative of the time delay $k$ of $L$ and the partial derivative is zero, the following equation for time delay is obtained as

$$
\frac{\partial L_{c}}{\partial k}+\sum_{j=1}^{2} \frac{\partial L_{j}}{\partial k}=0
$$

The solution of the equation is the maximum likelihood estimation value of time delay. But equation (13) is difficult to express as an explicit equation for time delay $k$. In order to facilitate the calculation of time delay, we turn to solve the following optimization problem

$$
\hat{k}=\arg \max _{k}\left(L_{c}+\sum_{j=1}^{2} L_{j}\right)
$$

When the sample are sufficient, the value of the second term of the objective function of the optimization problem (14) fluctuates little with the change of point $k$. In extreme cases, there is no obvious knowledge of $X_{1}, X_{2}$, we can think that $k$ is uniformly distributed. The value of the second term of the objective function of optimization problem (14) does not change with the change of point $k$. The objective function of the optimization problem is simplified as

$$
\hat{k}=\arg \max _{k}\left(L_{c}\right)
$$

At this point, the solution of the optimization problem to satisfy the equation $\mathrm{x}_{1}{ }^{i}=g\left(\mathrm{x}_{2}{ }^{i+k}\right)$ is $\hat{k}$.

Therefore, the time delay between random variables can be calculated using equation (15).

\section{SimULATION}

\section{A. First Order Linear System}

Consider the following first order linear continuous-time SISO system with a time delay, Simulink simulation block diagram is as follows

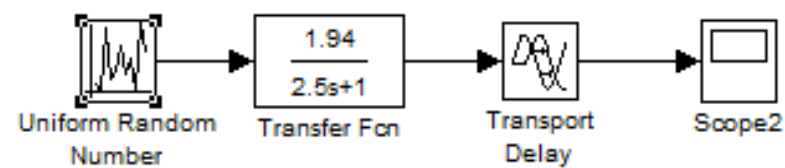

FIGURE I. FIRST ORDER LINEAR SYSTEM

The model of first order inertial time delay system is as follows

$$
G(s)=\frac{K e^{-\tau s}}{T s+1}
$$

where $\tau$ is the time delay parameter to be estimated. In order to further verify the correctness and applicability of the above method, where the two different relatively large parameters are selected to fix model. The first group parameters take $\mathrm{K}=$ $1.94, \mathrm{~T}=0.5, \tau=20$, and the second take $\mathrm{K}=9.4, \mathrm{~T}=$ $4.2, \tau=30$.
Using MATLAB programming to achieve the above method, the time delay parameter of the model is estimated. The specific estimation process can be simply described as: the first is that the data are collected, in which the input is the uniformly distributed random signal, the output is the output of the model and the sampling period is $1 \mathrm{~s}$; Then adopts the above equation (15) to estimate the time delay parameter of the model via the collected data.

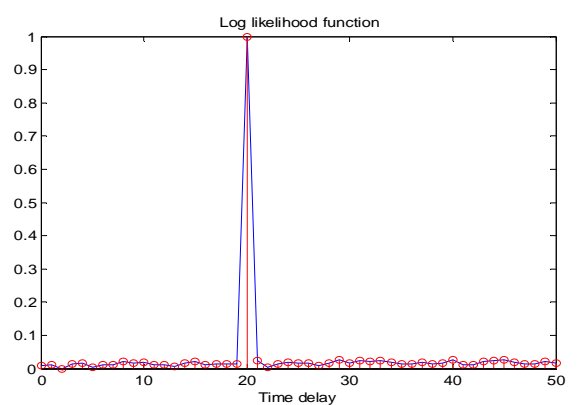

(A) TIME DELAY ESTIMATION RESULT $(\tau=20)$

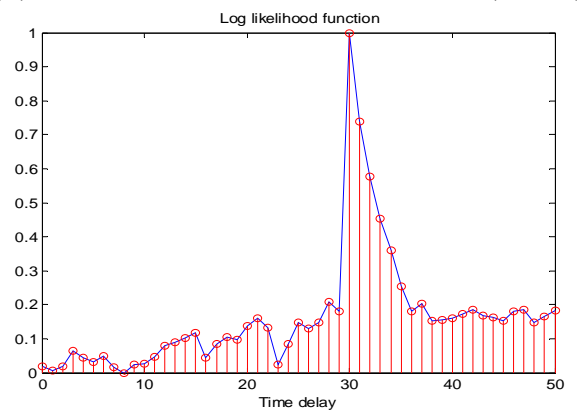

(B) TIME DELAY ESTIMATION RESULT $(\tau=30)$

FIGURE II. OUR METHOD FOR TIME DELAY ESTIMATION

Fig. 2 shows the log likelihood estimation of input and output for different delay times. In Fig. 2(A), the maximum value of the log likelihood function is $20 \mathrm{~s}$, and the value of the $\log$ likelihood function is smaller when the time delay is less than 20s and greater than 20s; In Fig. 2(B), the maximum value of the $\log$ likelihood function is $30 \mathrm{~s}$, and the value of the $\log$ likelihood function is smaller when the time delay is less than 30 s and greater than 30s. As shown in Fig. 2, the time delay is 20s in Fig. 2(A) and 30s in Fig. 2(B). The obtained of time delay value is consistent with the time delay parameter value selected in $G(s)$, which proves the effectiveness of the proposed method.

Comparison to other time delay identification methods in Fig. 3: Our method (A), Exhaustive method (B), PSO method (C) and System Identification Toolbox (Process models) (D). Where the value of parameters in $\mathrm{G}(\mathrm{s})$ is $\mathrm{K}=1.94, \mathrm{~T}=$ $2.5, \tau=20$. 


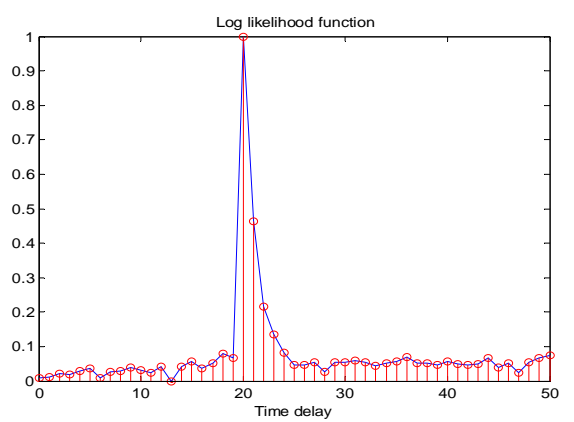

(A) OUR METHOD

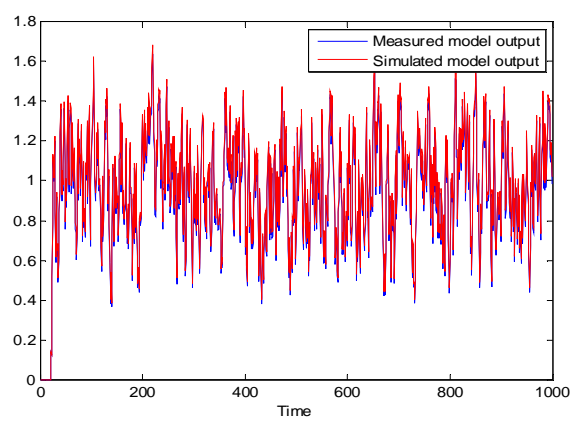

(B) EXHAUSTIVE METHOD

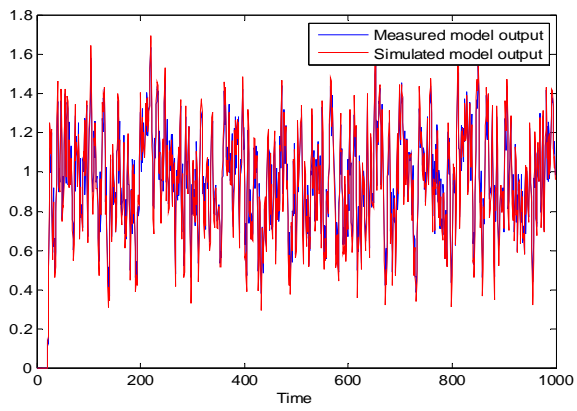

(C) PSO METHOD

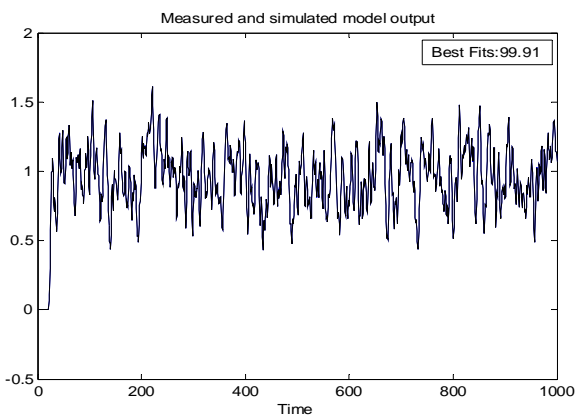

(D)SYSTEM IDENTIFICATION TOOLBOX (PROCESS MODELS) FIGURE III. FIRST ORDER LINEAR SYSTEM IDENTIFICATION

Fig. 3(B)-(D) depict measured data and identification result with exhaustive, PSO and System Identification Toolbox (Process models) methods. The time delay parameter identification results are summarized in Table 1.
TABLE I. TABLE ICOMPARISON TO OTHER METHODS IN FIRST ORDER LINEAR SYSTEM.

\begin{tabular}{ccl}
\hline Methods & Time delay estimation & error \\
\hline Our method & 20 & 0 \\
Exhaustive method & 20 & 0 \\
PSO method & 20.2159 & 0.0108 \\
System Identification & 20 & 0 \\
Toolbox (Process models) & & \\
\hline
\end{tabular}

\section{B. First Order Linear System with Noise}

Consider the case of noise in first order linear system, Simulink simulation block diagram is as follows

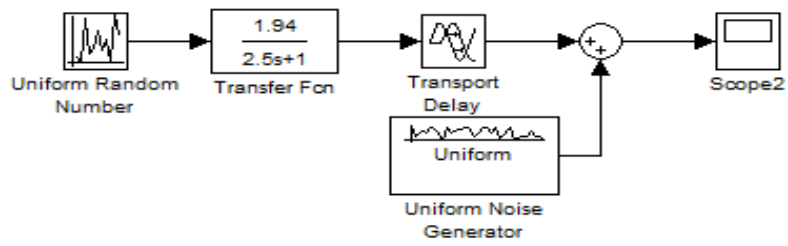

FIGURE IV. LINEAR SYSTEMS WITH NOISE

where the value of parameters in $\mathrm{G}(\mathrm{s})$ is $\mathrm{K}=1.94, \mathrm{~T}=$ $2.5, \tau=20, \tau$ is the time delay parameter to be estimated.

Comparison to other time delay identification methods in Fig. 5: Our method (A), Exhaustive method (B), PSO method (C) and System Identification Toolbox (Process models) (D).

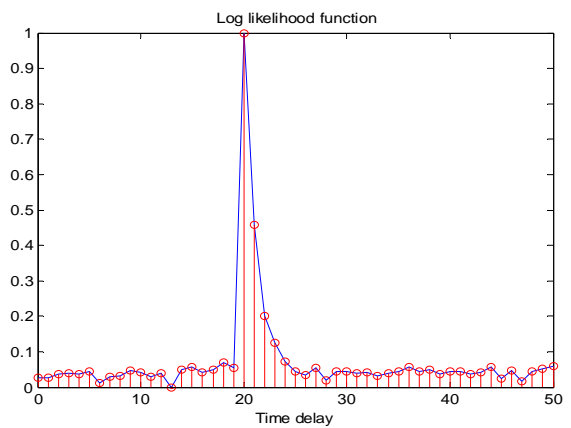

(A) OUR METHOD

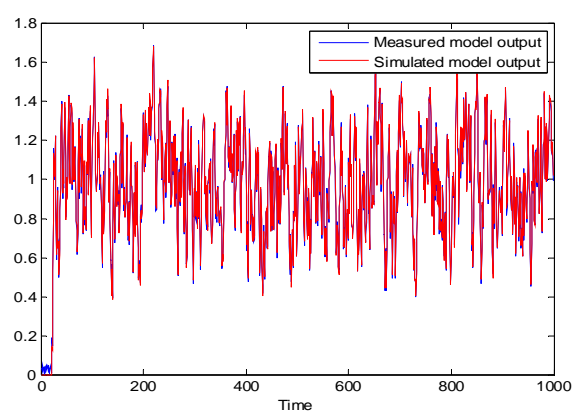

(B) EXHAUSTIVE METHOD 


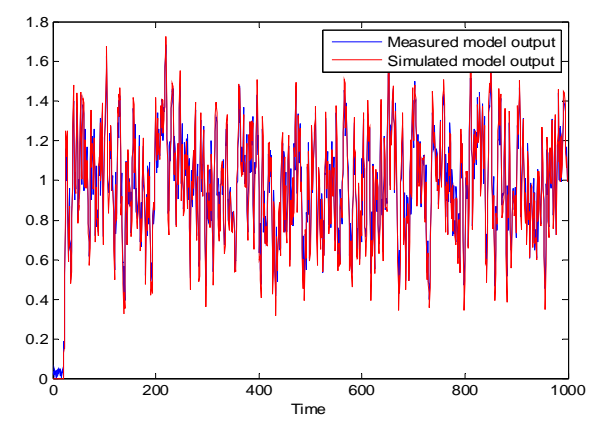

(C)PSO METHOD

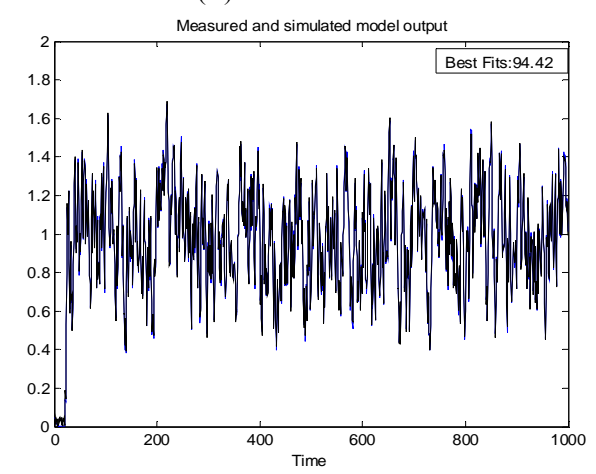

(D)SYSTEM IDENTIFICATION TOOLBOX (PROCESS MODELS)

FIGURE V. FIRST ORDER LINEAR SYSTEM WITH NOISE IDENTIFICATION

Fig. 5(B)-(D) depict measured data and identification result with exhaustive, PSO and System Identification Toolbox (Process models) methods. The time delay parameter identification results are summarized in Table 2.

TABLE II. COMPARISON TO OTHER METHODS IN FIRST ORDER LINEAR SYSTEM WITH NOISE.

\begin{tabular}{lcl}
\hline \multicolumn{1}{c}{ Methods } & Time delay estimation & error \\
\hline Our method & 20 & 0 \\
Exhaustive method & 20 & 0 \\
PSO method & 20.4705 & 0.0235 \\
$\begin{array}{l}\text { System Identification } \\
\text { Toolbox (Process models) }\end{array}$ & 19.992 & 0.0004 \\
\hline
\end{tabular}

From Table 1-2 we can find that our proposed estimation method obtained best time delay precision and error performance.

\section{CONCLUSION}

This paper is concerned with the time delay parameter estimation of first order linear SISO time delay systems. The time delay parameter is estimated by the maximum loglikelihood function of time delay. For the linear delay system identification, the good statistical properties of the time delay parameter can be obtained and the influence of noise disturbance is small, which proves the effectiveness of the proposed method. This method is based entirely on the input and output data of system, which is more practical for the complex systems in industry. When the time delay parameter is estimated, the input signal needs to have a rich change in the model. Future work will focus on extending the proposed idea for MIMO and nonlinear time delay systems.

\section{ACKNOWLEDGMENT}

The author would like to thank the anonymous reviewers and editors for the constructive comments that helped to improve this paper.

\section{REFERENCES}

[1] B. Zhou, ZY. Li, WX. Zheng, GR. Duan, "Stabilization of some linear systems with both state and input delays," Systems \& Control Letters , vol. 61, pp. 989-998, 2012.

[2] A. H. Quazi, "An overview on the time delay estimate in active and passive systems for target localization," IEEE Transactions on Acoustics, Speech and Signal Processing, vol. 29, pp. 527-533, June 1981.

[3] H. Kurz and W. Goedecke, "Digital parameter-adaptive control of processeswith unknown dead time," Automatica, vol. 17, pp.245-252, January1981.

[4] G. C. Carter, "Coherence and time delay estimation : an applied tutorial for research, development, test, and evaluation engineers," 1993.

[5] A. J. Isaksson, A. Horch, and G. A. Dumont, "Event-triggered deadtime estimation - comparison of methods." Technical report, Department of Signals, Sensors \& Systems, Royal Institute of Technology, Stockholm, Sweden, May 2001.

[6] S. Björklund, and L. Ljung, "An improved phase method for time-delay estimation ir." Automatica, vol. 45, 2013, pp.2467-2470.

[7] L. Ljung. System Identification: Theory for the User. Prentice-Hall, Upper Saddle River, N.J. USA, 2nd edition, 1999.

[8] L. Ljung, "Identification for control: Simple process models." In Proceedings of the 41st IEEE Conference on Decision and Control, pp. 4652-4657, Las Vegas, Nevada, USA, December 2002.

[9] P. Nagy and L. Ljung, "Estimating time-delays via state-space identification methods," In Preprints 9th IFAC Symposium on System Identification and System Parameter Estimation, pp.1141-1144, Budapest Hungary, Jul 1991.

[10] A. Elnaggar, G. A. Dumont, and A.-L. Elshafei, "Recursive estimation for system of unknown delay," In Proceedings of the 28th Conference on Decision and Control, Tampa, Florida, USA, December 1989.

[11] A. Ingimundarson, "Dead-Time Compensation and Performance Monitoring in Process Control." Phd thesis, Dep. of Automatic Control, Lund Institute of Technology, Lund, Sweden, 2003.

[12] C. L. Nikias and R. Pan, "Time delay estimation in unknown gaussianspatially correlated noise," IEEE Transactions on Acoustics, Speech,and Signal Processing, vol. 36, pp. 1706-1714, November 1988.

[13] H. Joe, "Multivariate models and multivariate dependence concepts," CRC Press, 1997.

[14] R B.Nelsen, “An introduction to copulas," Springer Science \& Business Media, 2013.

[15] M. Sklar, "Fonctions de répartition à n dimensions et leurs marges," Université Paris 8, 1959.

[16] Roch, Oriol, Alegre and Antonio, "Testing the bivariate distribution of daily equity returns using copulas. An application to the Spanish stock market." Computational Statistics \& Data Analysis, vol. 51, pp.13121329, 2005.

[17] R. D. Silva, and H. F. Lopes, "Copula, marginal distributions and model selection: a Bayesian note," Statistics and Computing, vol. 18, 2008, pp. 313-320.

[18] B. Choroś, R. Ibragimov, and E. Permiakova, Copula Estimation. Copula Theory and Its Applications. Springer Berlin Heidelberg, 2010, pp. 7791.

[19] F. Durante, and C. Sempi. Copula Theory: An Introduction. Copula Theory and Its Applications. 2010, pp. 3-31.

[20] R. Zhang, C. Czado, and A. Min, "Efficient maximum likelihood estimation of copula based meta t-distributions," Computational Statistics \& Data Analysis, vol. 55, 2011,pp. 1196-1214. 
[21] J .Dhaene, D. Linders, W. Schoutens, D. Vyncke, "A multivariate dependence measure for aggregating risks," Journal of Computational \& Applied Mathematics, vol. 263, 2014, pp. 78-87.

[22] P. H. S. Torr, "Bayesian Model Estimation and Selection for Epipolar Geometry and Generic Manifold Fitting," International Journal of Computer Vision, vol. 50, 2002, pp. 35-61.

[23] T. H. Lee, and X. Long. "Copula-based multivariate GARCH model with uncorrelated dependent errors," Journal of Econometrics, vol. 150, 2009, pp. $207-218$ 